\title{
Extreme blazars under the eyes of MAGIC
}

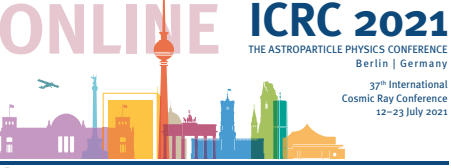

\section{A. Arbet-Engels, ${ }^{a, *}$ E. Prandini, ${ }^{b}$ S. Ventura, ${ }^{c}$ V. Fallah Ramazani, ${ }^{d}$ M. Cerruti, ${ }^{f}$ C. Arcaro, ${ }^{g}$ K. Asano, ${ }^{h}$ D. Dorner, ${ }^{i}$ M. Manganaro, ${ }^{j}$ G. Bonnoli, ${ }^{k, c}$ F. D'Ammando, ${ }^{l}$ L. Foffano, ${ }^{m}$ F. Tavecchio, ${ }^{g}$ J. Becerra-Gonzalez, ${ }^{n}$ J. Jormanainen ${ }^{d}$ and P. Da Vela ${ }^{o}$ on behalf of the MAGIC and FACT Collaborations}

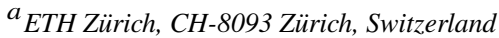

${ }^{b}$ Università di Padova and INFN, I-35131 Padova, Italy

${ }^{c}$ Università di Siena and INFN Pisa, I-53100 Siena, Italy

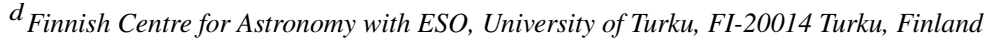

${ }^{e}$ Ruhr-Universität Bochum, 44801 Bochum, Germany

${ }^{f}$ Universitat de Barcelona, ICCUB, IEEC-UB, E-08028 Barcelona, Spain

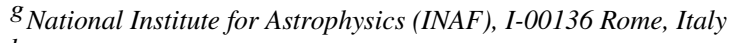

${ }^{h}$ Institute for Cosmic Ray Research (ICRR), The University of Tokyo, Kashiwa, 277-8582 Chiba, Japan

${ }^{i}$ Universität Würzburg, D-97074 Würzburg, Germany

${ }^{j}$ University of Rijeka, Department of Physics, 51000 Rijeka, Croatia

${ }^{k}$ Instituto de Astrofisica de Andalucia (CSIC), Apartado 3004, E-18080 Granada, Spain

${ }^{l}$ INAF - Istituto di Radioastronomia, Via Gobetti 101, I-40129 Bologna, Italy

${ }^{m}$ University of Geneva, Département de physique nucléaire et corpusculaire, 1211 Genève, Switzerland

${ }^{n}$ Instituto de Astrofísica de Canarias and Dpto. de Astrofísica, Universidad de La Laguna, 38200, La Laguna, Tenerife, Spain

${ }^{o}$ University of Innsbruck, Institute for Astro and Particle Physics, 6020 Innsbruck, Austria

E-mail: aaxel@phys.ethz.ch

Extreme high-frequency-peaked BL Lac objects (EHBLs) are the most energetic persistent sources in the universe. This contribution reports on long-term observing campaigns of tens of EHBLs that have been organized by the MAGIC collaboration to enlarge their population at VHE and understand the origin of their extreme properties. EHBLs are characterized by a spectral energy distribution (SED) featuring a synchrotron peak energy above $1 \mathrm{keV}$. Several EHBLs display a hard spectral index at very high energies (VHE; $E>100 \mathrm{GeV}$ ), suggesting a gamma-ray SED component peaking significantly above $1 \mathrm{TeV}$. Such extreme properties are challenging current standard emission and acceleration mechanisms. Recent studies have also unveiled intriguing disparities in the temporal characteristics of EHBLs. Some sources seem to display a persistent EHBL behaviour, while others belong to the EHBL family only temporarily. Here, we present recent results of the first hard-TeV EHBL catalog. The MAGIC observations are accompanied by an extensive multiwavelength coverage to obtain an optimal determination of the SED. This allow us to investigate leptonic and hadronic scenarios for the emission. We also present the recent detection of the EHBL RX J0812.0+0237 in the VHE band by MAGIC. Finally, we discuss a broad multiwavelength campaign on the BL Lac type object 1ES 2344+514, which showed intermittent EHBL characteristics in August 2016.

$37^{\text {th }}$ International Cosmic Ray Conference (ICRC 2021)

July 12 th - 23rd, 2021

Online - Berlin, Germany

\section{${ }^{*}$ Presenter}

$\dagger$ a complete list of the authors can be found at the end of the proceedings 


\section{Introduction}

Blazars form the largest class of sources in the extragalactic very-high-energy (VHE; $E>$ $100 \mathrm{GeV}$ ) sky ${ }^{1}$. They belong to the group of active galactic nuclei (AGN) and are identified by a relativistic plasma jet whose axis is aligned with the observer's line of sight [1]. The spectral energy distribution (SED) of blazars display two emission components: a low-energy component peaking in optical to X-ray energies and a high-energy component peaking in the gamma-ray band. It is now widely assumed that the low-energy component is emitted via synchrotron emission by relativistic electrons in the jet [2]. The origin of the high-energy component is still under debate and may be emitted by a leptonic and/or a hadronic particle population [2].

In the past decades, X-ray observations have revealed the existence of a blazar population with a low-energy component peaking at atypically high energies, above $1 \mathrm{keV} \mathrm{[3,4].} \mathrm{Blazars} \mathrm{with} \mathrm{such}$ extreme properties are dubbed as Extreme high-frequency-peaked BL Lac objects (EHBLs). Due to their low-energy SED component shifted to higher energies, EHBLs are identified by a hard X-ray spectrum with a photon index $\Gamma<2$. Some EHBLs also exhibit a high-energy SED component peaking above $1 \mathrm{TeV}$. The latter sources, dubbed as hard-TeV blazars, display VHE spectra with power-law indices $\Gamma \lesssim 2$.

The extreme properties EHBLs make them ideal targets to investigate emission and acceleration processes in blazar jets. To reproduce their SEDs, existing theoretical models are often challenged and require extreme parameter values $[4,5]$. EHBLs can also be used as cosmological probes. In particular, hard-TeV EHBLs give the opportunity to set limit on the strength of the intergalactic magnetic field (IGMF) by constraining the production of cascades triggered by $\sim 1 \mathrm{TeV}$ photons propagating through the universe $[6,7]$. With a spectrum extending significantly above $1 \mathrm{TeV}$, hard-TeV EHBLs are also powerful tools to constrain the intensity of the extragalactic background light (EBL; [8]).

\section{The MAGIC hard-TeV EHBL catalog}

Only a few number of hard-TeV blazars have been detected up to now. Extending the hard-TeV blazar population is therefore crucial given that they are powerful probes of blazar jet physics and cosmology. With this goal in mind, the MAGIC Collaboration performs multi-year observing campaigns of promising targets. The MAGIC telescopes form a system of two 17-m diameter imaging atmospheric Cherenkov telescopes (IACTs) [9]. They are located at an altitude of $\approx 2200 \mathrm{~m}$ above sea level on the island of La Palma, in Spain. The MAGIC telescopes are sensitive to photons at gamma-ray energies, from $\approx 20 \mathrm{GeV}$ to $\approx 100 \mathrm{TeV}$.

In this contribution, results of MAGIC observations between 2010 and 2017 of 10 potential hard-TeV blazars are summarised. The reader is referred to [10] for a complete report on the results. The selection of the targets was made by examining the spectral properties in the radio, X-rays and gamma rays as well as the redshift. The final list of selected targets is shown in Table 1. A total of 262 hours of observations was collected and analysed. From the 10 sources, only 1ES 1426+428 was known to be a VHE emitter prior to the start of the MAGIC observing campaign. The catalog

${ }^{1}$ http://tevcat2.uchicago.edu/ 


\begin{tabular}{lccccc}
\hline \hline Source & Epochs & $\begin{array}{c}\text { Detection significance } \\
{[\sigma]}\end{array}$ & $\begin{array}{c}E_{t h r} \\
{[\mathrm{TeV}]}\end{array}$ & $\begin{array}{c}\text { Flux } \\
{\left[10^{-12} \mathrm{~cm}^{-2} \mathrm{~s}^{-1}\right]}\end{array}$ & $\Gamma_{V H E, \text { intr }}$ \\
\hline \hline TXS 0210+515 & $2015,2016,2017$ & 5.9 & 0.2 & $1.6 \pm 0.5$ & $1.6 \pm 0.3$ \\
TXS 0637-128 & 2017 & 1.7 & 0.3 & $<8.9$ & - \\
BZB J0809+3455 & 2015 & 0.4 & 0.15 & $<3.7$ & - \\
RBS 0723 & 2013,2014 & 5.4 & 0.2 & $2.6 \pm 0.5$ & $2.7 \pm 1.2$ \\
1ES 0927+500 & 2012,2013 & 1.2 & 0.15 & $<5.1$ & - \\
RBS 0921 & 2016 & -0.4 & 0.15 & $<8.6$ & - \\
& 2010 & 2.1 & 0.2 & $<9.3$ & - \\
1ES 1426+428 & 2012 & 6.0 & 0.2 & $6.1 \pm 1.1$ & $1.8 \pm 0.5$ \\
& 2013 & 1.8 & 0.2 & $<5.1$ & - \\
1ES 2037+521 & 2016 & 7.5 & 0.3 & $1.8 \pm 0.4$ & $2.0 \pm 0.5$ \\
RGB J2042+244 & 2015 & 3.7 & 0.2 & $1.9 \pm 0.5$ & $1.7 \pm 0.6$ \\
RGB J2313+147 & 2015 & -0.9 & 0.2 & $<1.5$ & - \\
\hline 1ES 0229+200 & $2013-2017$ & 9.0 & 0.2 & $2.1 \pm 0.3$ & $1.8 \pm 0.1$ \\
\hline
\end{tabular}

Table 1: Sample of EHBLs observed by MAGIC between 2010 and 2017. From the list, only 1ES 1426+428 was a known VHE emitter prior to the MAGIC observing campaign; 1ES 0229+200 is used as a reference source. The second column gives the epochs of observations and the third column gives the detection significance (in units of $\sigma$ ). The fifth column shows the flux above the energy threshold $E_{t h r}$ given in the fourth column. An upper limit at $95 \%$ confidence level is quoted if no signal (or hint of a signal) is obtained. The last column is the index of the power-law fits performed for the sources with a signal (or hint of a signal).

of targets also includes the archetypal hard-TeV EHBL 1ES 0229+200, which is used as a reference source.

Table 1 lists the period of MAGIC observations for each source. The obtained detection significance is given in the third column. A significance above $5 \sigma$ was obtained for three sources previously undetected at VHE: TXS $0210+515$, RBS 0723 and 1ES 2037+521. Furthermore, a strong hint of signal $(\approx 4 \sigma)$ is obtained for RGB J2042+244. Regarding 1ES 1426+428, the source was also detected $(6 \sigma)$ in 2012, while observations in 2010 and 2013 yielded no significant signal. Table 1 also gives the corresponding VHE fluxes. They were computed above the energy threshold $E_{t h r}$ given in the fourth column. In case the source was detected below a significance of $3 \sigma$, an upper limit at $95 \%$ confidence level is quoted.

The last column of Table 1 shows the spectral index $\Gamma_{V H E \text {,intr }}$ obtained from a power-law fit $\left(d N / d E \propto E^{-\Gamma_{V H E, \text { intr }}}\right)$ to the sources that yielded a significant detection. The spectrum was also extracted for RGB J2042+244, whose observations yield a strong hint of a signal. The spectral index is the intrinsic one, i.e., the effect of the EBL absorption was corrected using the model template of [11]. All the indices are hard, they show best-fit values $\Gamma_{V H E \text {,intr }} \lesssim 2$, with the exception of RBS 0723, which best-fit index is softer than 2. TXS 0210+515 is the hardest source of the sample with a corresponding index significantly below $2, \Gamma_{V H E, \text { intr }}=1.6 \pm 0.3$. This indicates a highenergy SED component peaking above $1 \mathrm{TeV}$. Our study therefore firmly establishes TXS 0210+515 as a new member of the hard-TeV blazar family. The other sources (1ES 1426+428, 1ES 2037+521 and RGB J2042+244) remain compatible with a hard-TeV behaviour.

In order to characterise the synchrotron emission component, the MAGIC observations were complemented by a simultaneous X-ray coverage provided by the X-ray Telescope (XRT) on-board the Neil Gehrels Swift Observatory (Swift). Moreover, the Nuclear Spectroscopic Telescope Array 

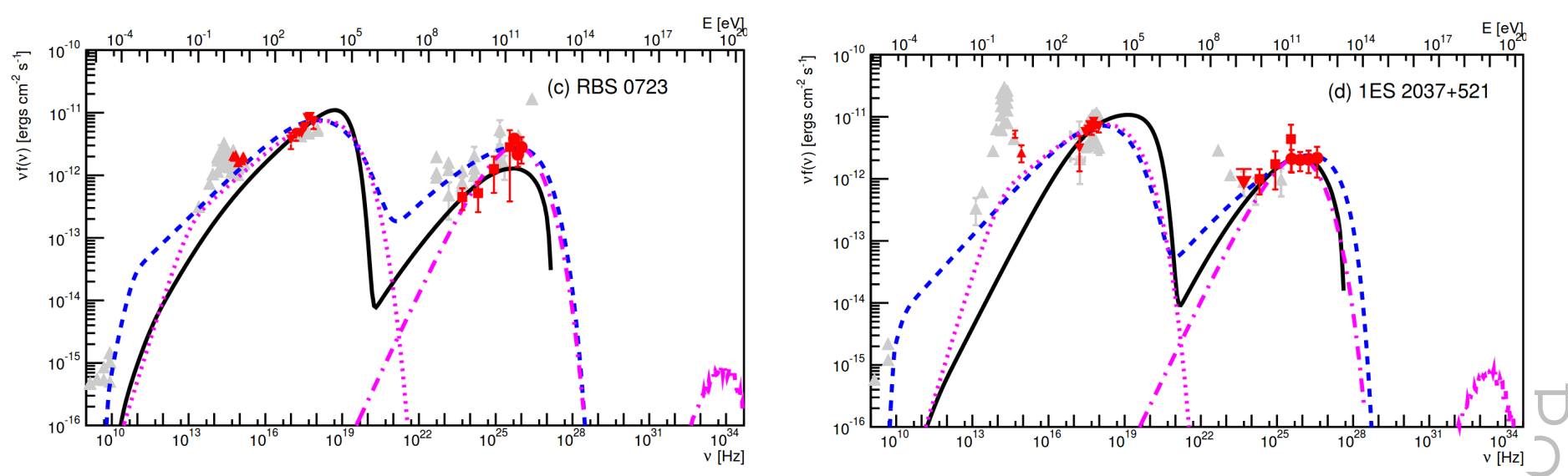

Figure 1: Broadband SED for RBS 0723 (left) and 1ES 2037+521 (right). Red data points are the quasisimultaneous measurements during the MAGIC observing campaign, while grey data points are archival data. The blue dashed curves show the one-zone SSC model applied to the data, the black continuous lines show the result of the spine-layer model and dashed-dotted magenta lines are the proton-synchrotron model. The magenta curves at $10^{33}-10^{34} \mathrm{~Hz}$ is the neutrino output from the proton-synchrotron model. The plots are taken from [10].

(NuSTAR) observed TXS $0210+515$ and RGB J2313+147. While XRT is covering the $0.3-10 \mathrm{keV}$ band, NUSTAR is observing in the $3-79 \mathrm{keV}$ band. The combination of the two instruments offers an excellent coverage up to the hard X-rays, which is crucial to constrain the synchrotron SED of EHBLs that is peaking deep in the X-ray regime. From the Swift-XRT analysis, the $0.3-10 \mathrm{keV}$ spectra are almost all well fitted using a power-law function $d N / d E \propto E^{-\Gamma}$ with an index $\Gamma \lesssim 2$. This suggests a synchrotron peak frequency located around or above $1 \mathrm{keV}$. Only RGB J2313+147 showed an X-ray spectrum with a power-law index softer than 2, indicating that the synchrotron peak frequency was below $1 \mathrm{keV}$ and that the source did not behave as an EHBL during the observations. Overall, a clear harder-when-brighter behaviour was identified for each source, which is a common feature of BL Lac type objects [12-14].

The multiwavelength data allowed us to interpret the broadband SEDs within leptonic and hadronic scenarios. As a first leptonic model, we used the one-zone synchrotron self-Compton (SSC) model developed by [15], in which the emitting zone filled with relativistic electrons is moving downstream in a conical jet structure. As a second leptonic scenario, we used the spinelayer model from [16, 17]. The latter scenario assumes a stratified electron plasma jet composed of a central part, the spine, surrounded by a layer moving at a slower speed. Because of their relative motions, the emission of one region as observed in the frame of the other is seen boosted. This boosted emission brings an additional target photon field to each region and their respective inverse Compton emission are therefore enhanced. As discussed by [18], the main advantage of the spine-layer model is its ability to possibly solve the low magnetisation and equipartition issue often encountered in typical one-zone SSC models. Finally, we considered a proton-synchrotron model described in [19]. In this model, the low-energy SED still comes from electron synchrotron emission, while the high-energy SED component is emitted via proton-synchrotron radiation.

We show in Fig. 1 the results of the three emission models applied to two of the newly 


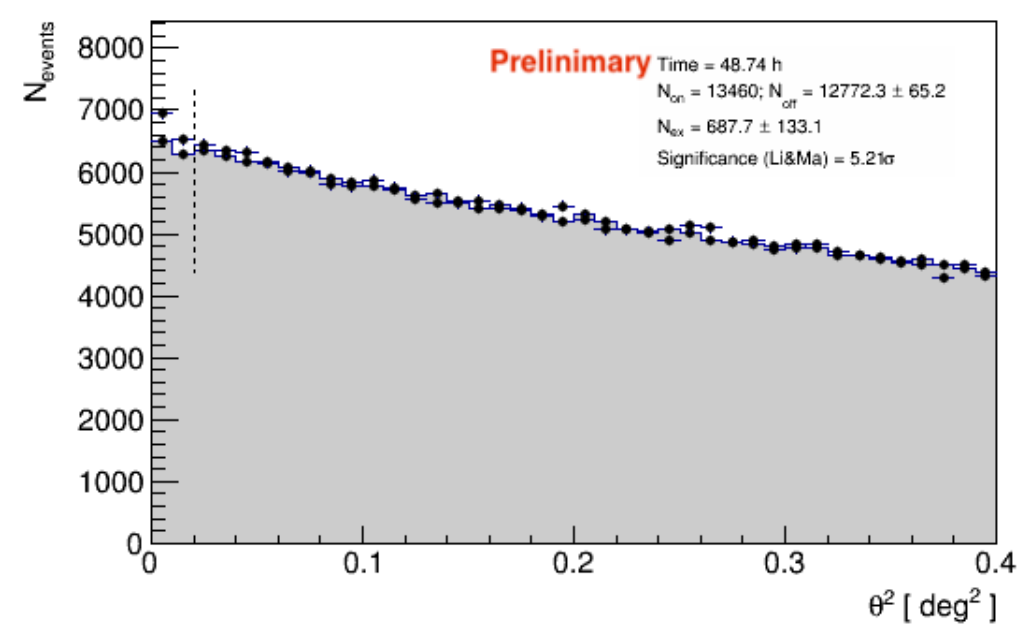

Figure 2: The $\theta^{2}$ distribution of RX J0812.0+0237. $\theta$ is the angular distance between the reconstructed event direction and the nominal source position. The grey histogram is the background distribution of $\theta^{2}$. The vertical dashed line defines the signal region below which the detection significance is computed.

VHE-detected sources from Table 1, RBS 0723 and 1ES 2037+521. The one-zone SSC model is represented with a blue dashed curve, the spine-layer model is plotted with a black continuous line and the proton-synchrotron model is plotted with a dashed-dotted magenta line. The reader is referred to [10] for the modelling results of the rest of the sources listed in Table 1. The three theoretical models are able to satisfactorily describe the observational data from all sources. Nevertheless, the parameters describing the emitting zone environment significantly differ between the three scenarios. We find that the one-zone SSC models always require a very low magnetisation of the emitting zone, leading to a system that is heavily out of equipartition. The magnetic field energy density $\left(U_{B}\right)$ is several orders of magnitude below the particle energy density $\left(U_{e}\right)$. One finds $U_{B} / U_{e} \sim 10^{-2}-10^{-4}$. Regarding the spine-layer model, we obtain solutions that can accommodate a region close to equipartition, $U_{B} / U_{e} \sim 1$. Finally, in the proton-synchrotron model, the magnetic field is much larger than the one used in the two previous leptonic models in order to compensate for the lower efficiency of the synchrotron emission by protons. Thus, in the proton-synchrotron models the magnetic energy density highly dominates over that of the particles and the system is out of equipartition. We note that within our proton-synchrotron model, the neutrino flux originating from proton-photon interactions in the jet is low and remains below the sensitivity of current neutrino telescopes.

\section{MAGIC detection of RX J0812.0+0237}

RX J0812.0+0237 is a BL Lac type object located at a redshift of $z=0.172$ [20]. The host galaxy is clearly identifiable in its SED, suggesting that the synchrotron SED component is shifted to higher frequencies with respect to less energetic BL Lacs. The flux properties of RX J0812.0+0237 in the radio, X-ray and MeV-GeV bands also show similarities with 1ES 0229+200, the archetypal EHBL [21]. An archival measurement from ROSAT at $1 \mathrm{keV}$ indicated a synchrotron SED extending in the hard X-ray regime [22], clearly suggesting that RX J0812.0+0237 is an EHBL. The source is listed in the fourth Fermi Large Area Telescope catalog (4FGL) [23], but remained undetected at VHE until now. 
MAGIC observed the source for a total of $\approx 49$ hours between 2019 and 2020 under good atmospheric conditions. Fig. 2 shows the resulting $\theta^{2}$ distribution, where $\theta$ is the angular distance between the reconstructed event direction and the nominal source position. The grey histogram shows the background distribution of $\theta^{2}$. The vertical dashed line defines the signal region below which the detection significance is computed. In the signal region, an excess of $N_{e x}=687.7 \pm 133.1$ gamma-ray events is obtained, corresponding to a detection significance of $5.21 \sigma$.

A preliminary MAGIC spectral analysis indicates that the intrinsic spectrum (i.e., corrected for the EBL absorption) in the VHE band is well described with a simple power-law function with a power-law index of $\Gamma_{V H E \text {,intr }}=2.58 \pm 0.33$.

The MAGIC observations were complemented by simultaneous data in the optical/UV and $\mathrm{X}$-rays by the Swift satellite to obtain a broadband view of the sources. The X-ray spectral analysis confirms the EHBL nature of the source, making it an ideal target to test particle acceleration models in blazar jets. A detailed investigation and interpretation of the broadband emission will be the topic of an upcoming publication.
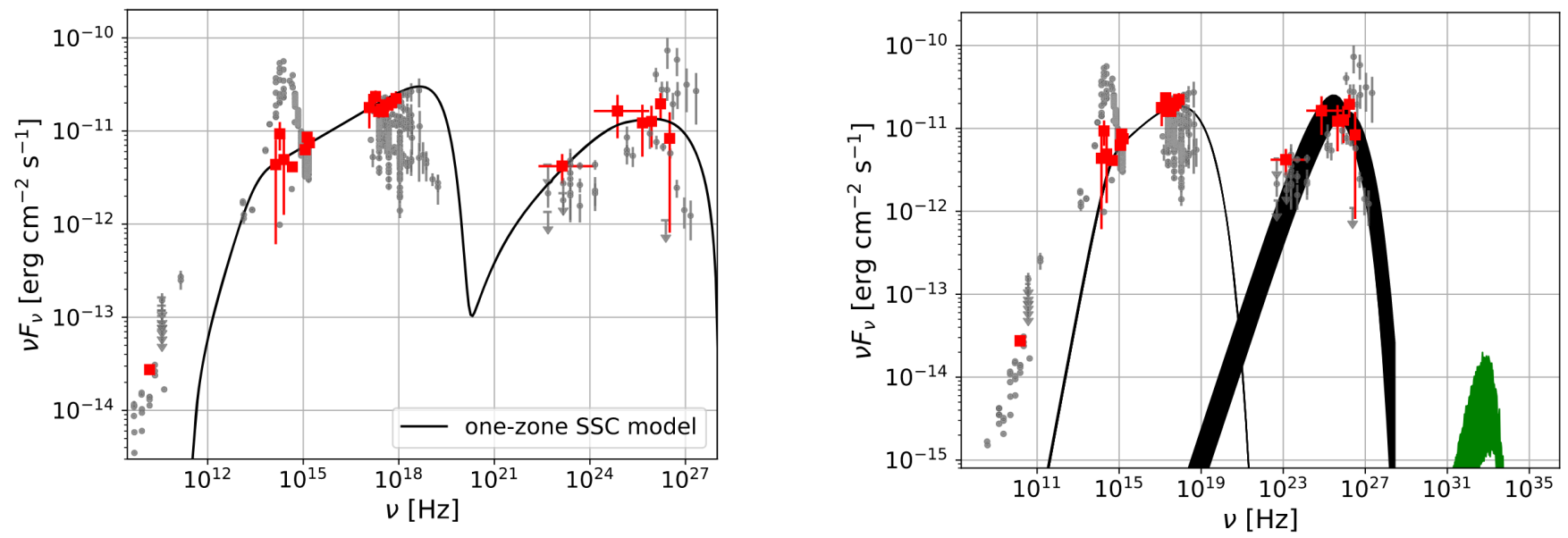

Figure 3: Broadband SED of 1ES 2344+514. Red data points show the observations during the VHE outburst in 2016, while grey data points are archival measurements. The black line on the left plot shows the results of the one-zone SSC model, while the black line on the right is the output of the proton-synchrotron model. The green curves at $10^{31}-10^{33} \mathrm{~Hz}$ are the neutrino flux arising from the proton-synchrotron model. The plots are taken from [25].

\section{The intermittent EHBL behaviour of 1ES 2344+514}

EHBLs come in different flavours: those showing a persistent EHBL behaviour and those showing EHBL characteristics only temporarily. The latter behaviour has been noticed for instance in Mrk 501 and 1ES 2344+514 [12, 24]. 1ES 2344+514 is a nearby BL Lac type object located at a redshift of $z=0.044$ [26]. It belongs to one of the first extragalactic sources detected at VHE. The first VHE detection was reported by the Whipple collaboration in 1995 during a flaring activity [27]. Observation from the BeppoSAX instrument during a high state in 1996 revealed X-ray $(0.1-10 \mathrm{keV})$ flux variability on hour time scale accompanied by strong spectral changes [24]. The synchrotron peak shifted to higher frequencies by a factor $\approx 30$, above $3 \times 10^{18} \mathrm{~Hz}(\approx 12 \mathrm{keV})$, which placed 
this source for the first time in the EHBL family. The recent multiwavelength campaigns occurred mostly during low activity, without the similar extremeness in the X-ray band seen by BeppoSAX in 1996. The extreme behaviour therefore seems to occur only during flaring states.

The First G-APD Cherenkov Telescope (FACT) is a 3.5-m diameter IACT located near the MAGIC telescopes, on the island of La Palma, in Spain [28, 29]. FACT is monitoring bright TeV blazars including 1ES 2344+514. On 2016 August 10, the FACT Quick Look Analysis [30], a lowlatency on-site analysis, detected the source in an enhanced state. The FACT collaboration issued an alert to the community, triggering MAGIC observations. MAGIC observed 1ES 2344+514 for two consecutive nights, 2016 August 11 and 2016 August 12, for a total of $\approx 1.1$ hours. During the first MAGIC observation the flux above $300 \mathrm{GeV}$ reached $F(>300 \mathrm{GeV})=(7.2 \pm 0.9) \times 10^{-11} \mathrm{~cm}^{-2} \mathrm{~s}^{-1}$, corresponding to about $60 \%$ of the flux of the Crab Nebula. This VHE flux level is similar to the historical maximum registered by Whipple in 1995 [27]. During the second MAGIC observation, the flux dropped by a factor $\approx 3.4$.

Several X-ray observations from Swift-XRT took place close in time to the MAGIC observations. The analysis reveals an enhanced $2-10 \mathrm{keV}$ flux around the VHE flare. The X-ray spectrum is well described by a simple power-law function with a hard index of $\Gamma=1.93 \pm 0.06$, indicating a synchrotron peak frequency above $1 \mathrm{keV}$. The latter value is about an order of magnitude higher than the one extracted during low-activity [31]. 1ES 2344+514 behaved temporarily as an EHBL during summer 2016.

We complemented the VHE and X-ray observations with radio, optical, infrared, UV and Fermi-LAT $(0.3-300 \mathrm{GeV})$ data in order to obtain an extensive multiwavelength characterisation of the source during this peculiar state. The combination of Fermi-LAT with VHE data from MAGIC and FACT provides an unprecedented constrain of the high-energy SED component of 1ES $2344+514$ during a flare. The broadband SED during the flare is shown Fig. 3 as red data points. At VHE, the spectrum is taken from the second MAGIC observing night (where the flux is $\approx 3.4$ times dimmer than the first observation) in order to be quasi-simultaneous to the Swift and other instruments observations. Grey data points show archival measurements from the SSDC (Space ScienceData Centre) database of ASI (Italian Space Agency) ${ }^{2}$. The strong shifts of the synchrotron peak frequency that $1 \mathrm{ES} 2344+514$ exhibits over time is clearly noticeable when comparing 2016 data with the archival measurements.

The unprecedented energy coverage during a flare of 1ES 2344+514 allowed us to test and constrain different models to interpret the broadband emission. We adopted a one-zone SSC model and a proton-synchrotron model from [19]. The obtained one-zone SSC model is represented with a black line in the left plot of Fig. 3, while the proton-synchrotron model is shown in black in the right plot. Both scenarios are able to well represent the observations. Nonetheless, the SSC model requires rather extreme parameters with electron population extending up to $\sim \mathrm{TeV}$ energies without strong cutoff and a very low magnetisation $\left(B \sim 10^{-2} \mathrm{G}\right)$. The system is several orders of magnitude out of equipartition, $U_{B} / U_{e} \sim 10^{-3}$. The opposite situation is found for the proton-synchrotron model in which the energy density of the magnetic field heavily dominates over the particle energy density. The green curve in the right plot of Fig. 3 shows the neutrino flux arising from protonphoton interactions, which is well below the sensitivity of current neutrino telescopes. The results

${ }^{2}$ http://www.asdc.asi.it/ 
of this work have been published in [25], where more details can be found.

\section{Conclusions}

EHBLs are powerful tools to study jet physics and can be used as cosmological probes. The MAGIC Collaboration set up an observing program in order to extend their population at VHE and to characterise their emission behaviour. The program has already proven to be a success, with several new EHBL detections, including hard-TeV blazars, which spectral properties represent a great challenge for current emission models. Complementing the MAGIC observation with dense multiwavelength coverage, we investigate different theoretical scenarios. Both leptonic and hadronic models are able to describe the observations at the cost of extreme parameters. Additional MAGIC observations of EHBLs are ongoing in order to build a second MAGIC hard-TeV blazar catalog.

\section{Acknowledgments}

The MAGIC Collaboration acknowledges the support from the agencies and organizations listed here:https://magic.mpp. mpg.de/acknowledgments_ICRC2021/. The FACT Collaboration acknowledges the support from the agencies and organizations listed here: https://fact-project.org/collaboration/icrc2021_acknowledgements.html

\section{References}

[1] Padovani P., Alexander D. M., Assef R. J., De Marco B., Giommi P., Hickox R. C., Richards G. T., et al., 2017 , A\&ARv, $25,2$.

[2] Böttcher M., 2007, Ap\&SS, 309, 95.

[3] Costamante L., Ghisellini G., Giommi P., Tagliaferri G., Celotti A., Chiaberge M., Fossati G., et al., 2001, A\&A, 371 , 512.

[4] Biteau J., Prandini E., Costamante L., Lemoine M., Padovani P., Pueschel E., Resconi E., et al., 2020, NatAs, 4, 124.

[5] Kaufmann S., Wagner S. J., Tibolla O., Hauser M., 2011, A\&A, 534, A130.

[6] H. E. S. S. Collaboration, Abramowski A., Aharonian F., Ait Benkhali F., Akhperjanian A. G., Angüner E., Anton G., et al., 2014, A\&A, 562, A145

[7] Archambault S., Archer A., Benbow W., Buchovecky M., Bugaev V., Cerruti M., Connolly M. P., et al., 2017 , ApJ, 835, 288.

[8] Aharonian F., Akhperjanian A. G., Bazer-Bachi A. R., Beilicke M., Benbow W., Berge D., Bernlöhr K., et al., 2006, Natur, 440, 1018.

[9] Aleksić J., Ansoldi S., Antonelli L. A., Antoranz P., Babic A., Bangale P., Barceló M., et al., 2016, APh, 72, 76.

[10] Acciari V. A., Ansoldi S., Antonelli L. A., Engels A. A., Asano K., Baack D., Babić A., et al., 2020, ApJS, $247,16$.

[11] Franceschini A., Rodighiero G., Vaccari M., 2008, A\&A, 487, 837.

[12] Pian E., Vacanti G., Tagliaferri G., Ghisellini G., Maraschi L., Treves A., Urry C. M., et al., 1998, ApJL, 492, L17.

[13] Krawczynski H., Hughes S. B., Horan D., Aharonian F., Aller M. F., Aller H., Boltwood P., et al., 2004 , ApJ, $601,151$.

[14] Acciari V. A., Ansoldi S., Antonelli L. A., Asano K., Babić A., Banerjee B., Baquero A., et al., 2021, MNRAS, $504,1427$.

[15] Asano K., Takahara F., Kusunose M., Toma K., Kakuwa J., 2014, ApJ, 780, 64.

[16] Ghisellini G., Tavecchio F., Chiaberge M., 2005, A\&A, 432, 401.

[17] Tavecchio F., Ghisellini G., 2008, MNRAS, 385, L98.

[18] Tavecchio F., Ghisellini G., 2016, MNRAS, 456, 2374.

[19] Cerruti M., Zech A., Boisson C., Inoue S., 2015, MNRAS, 448, 910.

[20] González J. B., Acosta-Pulido J. A., Boschin W., Clavero R., Otero-Santos J., Carballo-Bello J. A., Domínguez-Palmero L., 2021, MNRAS, 504, 5258.

[21] Tavecchio F., Ghisellini G., Ghirlanda G., Costamante L., Franceschini A., 2009, MNRAS, 399, L59.

[22] Voges W., Aschenbach B., Boller T., Bräuninger H., Briel U., Burkert W., Dennerl K., et al., 1999, A\&A, 349, 389

[23] Abdollahi S., Acero F., Ackermann M., Ajello M., Atwood W. B., Axelsson M., Baldini L., et al., 2020, ApJS, $247,33$.

[24] Giommi P., Padovani P., Perlman E., 2000, MNRAS, 317, 743.

[25] MAGIC Collaboration, Acciari V. A., Ansoldi S., Antonelli L. A., Arbet Engels A., Babić A., Banerjee B., et al., 2020, MNRAS, 496, 3912.

[26] Perlman E. S., Stocke J. T., Schachter J. F., Elvis M., Ellingson E., Urry C. M., Potter M., et al., 1996, ApJS, $104,251$.

[27] Catanese M., Akerlof C. W., Badran H. M., Biller S. D., Bond I. H., Boyle P. J., Bradbury S. M., et al., 1998, ApJ, $501,616$.

[28] Anderhub H., Backes M., Biland A., Boccone V., Braun I., Bretz T., Buß J., et al., 2013, JInst, 8, P06008.

[29] Biland A., Bretz T., Buß J., Commichau V., Djambazov L., Dorner D., Einecke S., et al., 2014, JInst, 9 , P10012.

[30] Dorner D., Ahnen M. L., Bergmann M., Biland A., Balbo M., Bretz T., Buss J., et al., 2015, arXiv, arXiv:1502.02582

[31] Nilsson K., Lindfors E., Takalo L. O., Reinthal R., Berdyugin A., Sillanpää A., Ciprini S., et al., 2018, A\&A, 620 , A185. 
A. Arbet-Engels

\section{Full Authors List:}

\section{MAGIC Collaboration:}

V. A. Acciari ${ }^{1}$, S. Ansoldi ${ }^{2,41}$, L. A. Antonelli ${ }^{3}$, A. Arbet-Engels ${ }^{4}$, M. Artero ${ }^{5}$, K. Asano ${ }^{6}$, D. Baack ${ }^{7}$, A. Babić ${ }^{8}$, A. Baquero ${ }^{9}$, U. Barres de Almeida ${ }^{10}$, J. A. Barrio ${ }^{9}$, I. Batković ${ }^{11}$, J. Becerra González ${ }^{1}$, W. Bednarek ${ }^{12}$, L. Bellizzi ${ }^{13}$, E. Bernardini ${ }^{14}$, M. Bernardos ${ }^{11}$, A. Berti ${ }^{15}$, J. Besenrieder ${ }^{15}$, W. Bhattacharyya ${ }^{14}$, C. Bigongiari ${ }^{3}$, A. Biland ${ }^{4}$, O. Blanch ${ }^{5}$, H. Bökenkamp ${ }^{7}$, G. Bonnoli ${ }^{16}$, Ž. Bošnjak $^{8}$, G. Busetto ${ }^{11}$, R. Carosi ${ }^{17}$, G. Ceribella ${ }^{15}$, M. Cerruti ${ }^{18}$, Y. Chai ${ }^{15}$, A. Chilingarian ${ }^{19}$, S. Cikota ${ }^{8}$, S. M. Colak ${ }^{5}$, E. Colombo ${ }^{1}$, J. L. Contreras ${ }^{9}$, J. Cortina ${ }^{20}$, S. Covino ${ }^{3}$, G. D’Amico ${ }^{15,42}$, V. D’Elia ${ }^{3}$, P. Da Vela ${ }^{17,43}$, F. Dazzi ${ }^{3}$, A. De Angelis ${ }^{11}$, B. De Lotto ${ }^{2}$, M. Delfino ${ }^{5,44}$, J. Delgado ${ }^{5,44}$, C. Delgado Mendez $^{20}$, D. Depaoli ${ }^{21}$, F. Di Pierro ${ }^{21}$, L. Di Venere ${ }^{22}$, E. Do Souto Espiñeira ${ }^{5}$, D. Dominis Prester $^{23}$, A. Donini ${ }^{2}$, D. Dorner ${ }^{24}$, M. Doro ${ }^{11}$, D. Elsaesser ${ }^{7}$, V. Fallah Ramazani ${ }^{25,45}$, A. Fattorini ${ }^{7}$, M. V. Fonseca ${ }^{9}$, L. Font ${ }^{26}$, C. Fruck ${ }^{15}$, S. Fukami ${ }^{6}$, Y. Fukazawa ${ }^{27}$, R. J. García López ${ }^{1}$, M. Garczarczyk ${ }^{14}$, S. Gasparyan ${ }^{28}$, M. Gaug ${ }^{26}$, N. Giglietto ${ }^{22}$, F. Giordano ${ }^{22}$, P. Gliwny ${ }^{12}$, N. Godinović ${ }^{29}$, J. G. Green ${ }^{3}$, D. Green ${ }^{15}$, D. Hadasch ${ }^{6}$, A. Hahn ${ }^{15}$, L. Heckmann ${ }^{15}$, J. Herrera ${ }^{1}$,

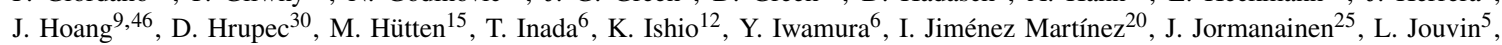
M. Karjalainen ${ }^{1}$, D. Kerszberg ${ }^{5}$, Y. Kobayashi' ${ }^{6}$, H. Kubo ${ }^{31}$, J. Kushida ${ }^{32}$, A. Lamastra ${ }^{3}$, D. Lelas ${ }^{29}$, F. Leone ${ }^{3}$, E. Lindfors ${ }^{25}$, L. Linhoff ${ }^{7}$, S. Lombardi ${ }^{3}$, F. Longo ${ }^{2,47}$, R. López-Coto ${ }^{11}$, M. López-Moya ${ }^{9}$, A. López-Oramas ${ }^{1}$, S. Loporchio ${ }^{22}$, B. Machado de Oliveira Fraga $^{10}$, C. Maggio ${ }^{26}$, P. Majumdar ${ }^{33}$, M. Makariev ${ }^{34}$, M. Mallamaci ${ }^{11}$, G. Maneva ${ }^{34}$, M. Manganaro ${ }^{23}$, K. Mannheim ${ }^{24}$, L. Maraschi ${ }^{3}$, M. Mariotti ${ }^{11}$, M. Martínez ${ }^{5}$, D. Mazin ${ }^{6,15}$, S. Menchiari ${ }^{13}$, S. Mender ${ }^{7}$, S. Mićanović2 ${ }^{23}$, D. Miceli ${ }^{2,49}$, T. Miener ${ }^{9}$, J. M. Miranda ${ }^{13}$, R. Mirzoyan ${ }^{15}$, E. Molina ${ }^{18}$, A. Moralejo ${ }^{5}$, D. Morcuende ${ }^{9}$, V. Moreno ${ }^{26}$, E. Moretti ${ }^{5}$, T. Nakamori ${ }^{35}$, L. Nava ${ }^{3}$, V. Neustroev ${ }^{36}$, C. Nigro $^{5}$, K. Nilsson ${ }^{25}$, K. Nishijima ${ }^{32}$, K. Noda $^{6}$, S. Nozaki ${ }^{31}$, Y. Ohtani ${ }^{6}$, T. Oka ${ }^{31}$, J. Otero-Santos ${ }^{1}$, S. Paiano ${ }^{3}$, M. Palatiello ${ }^{2}$, D. Paneque ${ }^{15}$, R. Paoletti ${ }^{13}$, J. M. Paredes ${ }^{18}$, L. Pavletić ${ }^{23}$, P. Peñil ${ }^{9}$, M. Persic ${ }^{2,50}$, M. Pihet ${ }^{15}$, P. G. Prada Moroni ${ }^{17}$, E. Prandini ${ }^{11}$, C. Priyadarshi ${ }^{5}$, I. Puljak ${ }^{29}$, W. Rhode ${ }^{7}$, M. Ribó ${ }^{18}$, J. Rico ${ }^{5}$, C. Righi ${ }^{3}$, A. Rugliancich ${ }^{17}$, N. Sahakyan ${ }^{28}$, T. Saito ${ }^{6}$, S. Sakurai ${ }^{6}$, K. Satalecka ${ }^{14}$, F. G. Saturni ${ }^{3}$, B. Schleicher ${ }^{24}$, K. Schmidt ${ }^{7}$, T. Schweizer ${ }^{15}$, J. Sitarek ${ }^{12}$, I. Šnidarić ${ }^{37}$, D. Sobczynska ${ }^{12}$, A. Spolon ${ }^{11}$, A. Stamerra ${ }^{3}$, J. Strišković ${ }^{30}$, D. Strom ${ }^{15}$, M. Strzys ${ }^{6}$, Y. Suda ${ }^{27}$, T. Surić ${ }^{37}$, M. Takahashi ${ }^{6}$, R. Takeishi ${ }^{6}$, F. Tavecchio ${ }^{3}$, P. Temnikov ${ }^{34}$, T. Terzic ć $^{23}$, M. Teshima ${ }^{15,6}$, L. Tosti ${ }^{38}$, S. Truzzi ${ }^{13}$, A. Tutone ${ }^{3}$, S. Ubach ${ }^{26}$, J. van Scherpenberg ${ }^{15}$, G. Vanzo ${ }^{1}$, M. Vazquez Acosta ${ }^{1}$, S. Ventura ${ }^{13}$, V. Verguilov ${ }^{34}$, C. F. Vigorito ${ }^{21}$, V. Vitale ${ }^{39}$, I. Vovk $^{6}$, M. Will ${ }^{15}$, C. Wunderlich ${ }^{13}$, T. Yamamoto ${ }^{40}$, and D. Zaric 29

${ }^{1}$ Instituto de Astrofísica de Canarias and Dpto. de Astrofísica, Universidad de La Laguna, E-38200, La Laguna, Tenerife, Spain ${ }^{2}$ Università di Udine and INFN Trieste, I-33100 Udine, Italy ${ }^{3}$ National Institute for Astrophysics (INAF), I-00136 Rome, Italy ${ }^{4}$ ETH Zürich, CH-8093 Zürich, Switzerland ${ }^{5}$ Institut de Física d'Altes Energies (IFAE), The Barcelona Institute of Science and Technology (BIST), E-08193 Bellaterra (Barcelona), Spain ${ }^{6}$ Japanese MAGIC Group: Institute for Cosmic Ray Research (ICRR), The University of Tokyo, Kashiwa, 277-8582 Chiba, Japan ${ }^{7}$ Technische Universität Dortmund, D-44221 Dortmund, Germany ${ }^{8}$ Croatian MAGIC Group: University of Zagreb, Faculty of Electrical Engineering and Computing (FER), 10000 Zagreb, Croatia ${ }^{9}$ IPARCOS Institute and EMFTEL Department, Universidad Complutense de Madrid, E-28040 Madrid, Spain ${ }^{10}$ Centro Brasileiro de Pesquisas Físicas (CBPF), 22290-180 URCA, Rio de Janeiro (RJ), Brazil ${ }^{11}$ Università di Padova and INFN, I-35131 Padova, Italy ${ }^{12}$ University of Lodz, Faculty of Physics and Applied Informatics, Department of Astrophysics, 90-236 Lodz, Poland ${ }^{13}$ Università di Siena and INFN Pisa, I-53100 Siena, Italy ${ }^{14}$ Deutsches Elektronen-Synchrotron (DESY), D-15738 Zeuthen, Germany ${ }^{15}$ Max-Planck-Institut für Physik, D-80805 München, Germany ${ }^{16}$ Instituto de Astrofísica de Andalucía-CSIC, Glorieta de la Astronomía s/n, 18008, Granada, Spain ${ }^{17}$ Università di Pisa and INFN Pisa, I-56126 Pisa, Italy ${ }^{18}$ Universitat de Barcelona, ICCUB, IEEC-UB, E-08028 Barcelona, Spain ${ }^{19}$ Armenian MAGIC Group: A. Alikhanyan National Science Laboratory, 0036 Yerevan, Armenia ${ }^{20}$ Centro de Investigaciones Energéticas, Medioambientales y Tecnológicas, E-28040 Madrid, Spain ${ }^{21}$ INFN MAGIC Group: INFN Sezione di Torino and Università degli Studi di Torino, I-10125 Torino, Italy ${ }^{22}$ INFN MAGIC Group: INFN Sezione di Bari and Dipartimento Interateneo di Fisica dell'Università e del Politecnico di Bari, I-70125 Bari, Italy ${ }^{23}$ Croatian MAGIC Group: University of Rijeka, Department of Physics, 51000 Rijeka, Croatia ${ }^{24}$ Universität Würzburg, D-97074 Würzburg, Germany ${ }^{25}$ Finnish MAGIC Group: Finnish Centre for Astronomy with ESO, University of Turku, FI-20014 Turku, Finland ${ }^{26}$ Departament de Física, and CERES-IEEC, Universitat Autònoma de Barcelona, E-08193 Bellaterra, Spain ${ }^{27}$ Japanese MAGIC Group: Physics Program, Graduate School of Advanced Science and Engineering, Hiroshima University, 739-8526 Hiroshima, Japan ${ }^{28}$ Armenian MAGIC Group: ICRANet-Armenia at NAS RA, 0019 Yerevan, Armenia ${ }^{29}$ Croatian MAGIC Group: University of Split, Faculty of Electrical Engineering, Mechanical Engineering and Naval Architecture (FESB), 21000 Split, Croatia ${ }^{30}$ Croatian MAGIC Group: Josip Juraj Strossmayer University of Osijek, Department of Physics, 31000 Osijek, Croatia ${ }^{31}$ Japanese MAGIC Group: Department of Physics, Kyoto University, 606-8502 Kyoto, Japan ${ }^{32}$ Japanese MAGIC Group: Department of Physics, Tokai University, Hiratsuka, 259-1292 Kanagawa, Japan ${ }^{33}$ Saha Institute of Nuclear Physics, HBNI, 1/AF Bidhannagar, Salt Lake, Sector-1, Kolkata 700064, India ${ }^{34}$ Inst. for Nucl. Research and Nucl. Energy, Bulgarian Academy of Sciences, BG-1784 Sofia, Bulgaria ${ }^{35}$ Japanese MAGIC Group: Department of Physics, Yamagata University, Yamagata 990-8560, Japan ${ }^{36}$ Finnish MAGIC Group: Astronomy Research Unit, University of Oulu, FI-90014 Oulu, Finland ${ }^{37}$ Croatian MAGIC Group: Ruđer Bošković Institute, 10000 Zagreb, Croatia ${ }^{38}$ INFN MAGIC Group: INFN Sezione di Perugia, I-06123 Perugia, Italy ${ }^{39}$ INFN MAGIC Group: INFN Roma Tor Vergata, I-00133 Roma, Italy ${ }^{40}$ Japanese MAGIC Group: Department of Physics, Konan University, Kobe, Hyogo 658-8501, Japan ${ }^{41}$ also at International Center for Relativistic Astrophysics (ICRA), Rome, Italy ${ }^{42}$ now at Department for Physics and Technology, University of Bergen, NO-5020, Norway ${ }^{43}$ now at University of Innsbruck ${ }^{44}$ also at Port d'Informació Científica (PIC), E-08193 Bellaterra (Barcelona), Spain ${ }^{45}$ now at Ruhr-Universität Bochum, Fakultät für Physik und Astronomie, Astronomisches Institut (AIRUB), 44801 Bochum, Germany ${ }^{46}$ now at Department of Astronomy, University of California Berkeley, Berkeley CA $94720^{47}$ also at Dipartimento di Fisica, Università di Trieste, I-34127 Trieste, Italy ${ }^{49}$ now at Laboratoire 
d'Annecy de Physique des Particules (LAPP), CNRS-IN2P3, 74941 Annecy Cedex, France ${ }^{50}$ also at INAF Trieste and Dept. of Physics and Astronomy, University of Bologna, Bologna, Italy

\section{FACT Collaboration:}

A. Arbet-Engels ${ }^{1}$, D. Baack ${ }^{2}$, M. Balbo ${ }^{3}$, A. Biland ${ }^{1}$, T. Bretz ${ }^{1, a}$, J. Buss ${ }^{2}$, D. Dorner ${ }^{4}$, L. Eisenberger ${ }^{4}$, D. Elsaesser ${ }^{2}$, D. Hildebrand ${ }^{1}$, R. Iotov ${ }^{4}$, A. Kalenski ${ }^{4}$, K. Mannheim ${ }^{4}$, A. Mitchell ${ }^{1}$, D. Neise ${ }^{1}$, M. Noethe ${ }^{2}$, A. Paravac ${ }^{4}$, W. Rhode ${ }^{2}$, B. Schleicher ${ }^{4}$, V. Sliusar ${ }^{3}$, R. Walter ${ }^{3}$

${ }^{1}$ ETH Zurich, Institute for Particle Physics and Astrophysics, Otto-Stern-Weg 5, 8093 Zurich, Switzerland ${ }^{2}$ TU Dortmund, Experimental Physics 5, Otto-Hahn-Str. 4a, 44227 Dortmund, Germany ${ }^{3}$ University of Geneva, Department of Astronomy, Chemin dÉcogia 16, 1290 Versoix, Switzerland ${ }^{4}$ University of Würzburg, Institute for Theoretical Physics and Astrophysics, Emil-Fischer-Str. 31, 97074 Würzburg, Germany ${ }^{a}$ also at RWTH Aachen University 